\title{
Opto-acoustic chemical sensor based on forward stimulated Brillouin scattering in optical fiber
}

\section{(Invited)}

\author{
Desmond M. Chow*, Luc Thévenaz \\ EPFL Swiss Federal Institute of Technology \\ Institute of Electrical Engineering \\ SCI-STI-LT, Station 11, \\ CH-1015 Lausanne, Switzerland \\ *desmond.chow@epfl.ch
}

\begin{abstract}
Stimulated Brillouin scattering is well-known for providing distributed measurements in the field of optical fiber sensing. For the conventional backward stimulated Brillouin scattering, the sensing capability is nevertheless limited to temperature and mechanical strain in the core of an optical fiber. Forward stimulated Brillouin scattering (FSBS), due to the participating transverse acoustic waves, can be used to directly measure the mechanical properties of the material that surrounds a standard telecom fiber. We review our work on harnessing FSBS to extract the acoustic impedances of different liquid materials. The acoustic impedance mismatch between silica optical fiber and its surroundings determines the decay rate or the corresponding resonance linewidth of the transverse acoustic modes. We address the measurement with both time and frequency domain techniques, demonstrating accuracy of measurements up to $95 \%$. This work could be potentially extended to realize fiber based ultrasonic chemical analysis.
\end{abstract}

Keywords—-forward stimulated Brillouin scattering, optical fiber sensors, acoustic impedance measurement.

\section{INTRODUCTION}

Stimulated Brillouin scattering in silica optical fiber is a well-studied opto-acoustic interaction that has been widely used in distributed fiber sensing [1]. However, for the conventional backward stimulated Brillouin scattering, the detection is limited to temperature and mechanical strain because the participating longitudinal acoustic wave is confined in the core of an optical fiber. Forward stimulated Brillouin scattering (FSBS), also known as guided acoustic waves Brillouin scattering (GAWBS) [2], is an opto-acoustic effect involving interaction of the guided light with the transverse acoustic eigenmodes in an optical waveguide, which can be stimulated via electrostriction. Recently, Antman et al. have demonstrated measurement of liquid acoustic impedance using FSBS [3]. By removing the acrylate coating of a standard single-mode fiber (SMF), transverse acoustic waves in the fiber cladding are allowed to interact directly with the fiber surroundings through acoustic reflection. The acoustic decay time or the corresponding resonance linewidth, both affected by the boundary reflectivity, could be retrieved to calculate the acoustic impedance of the surrounding liquid medium.
The FSBS response can be observed by measuring the refractive index perturbation caused by the induced transverse acoustic waves. For acoustic impedance measurement, similar results could be obtained by using either a time-domain or a frequency-domain measurement technique. For the time-domain technique [3], a high intensity short pulse approximating Dirac delta function is launched into the fiber to generate multiple acoustic modes and simultaneously an interferometer is used to convert optical phase changes in the fiber to intensity variations detectable by a photodiode. Subsequently, the decay time of a particular mode must be obtained by applying Fourier transform numerically to the temporal trace and spectrally selecting a specific resonance peak for analysis.

For the frequency domain technique [4], a continuous wave (CW) light is intensity-modulated at the FSBS resonance frequency to stimulate a specific transverse acoustic mode. The resonance spectrum is then obtained by sweeping the modulation frequency across the resonance frequency. Because of Kerr effect giving a similar signature in phase quadrature, the detected spectrum shows Fano resonance shape that makes the retrieval of the acoustic resonance linewidth challenging. Taking advantage of the contrast between the long tail of FSBS response and the instantaneous nature of Kerr effect, transverse acoustic wave is stimulated within a long pulse, yet only the acoustic response outside of the pulse duration is selectively gated for detection [4]. Using both time and frequency domain techniques, the acoustic impedances of water and ethanol are measured and agree with the reported standard values.

\section{PRINCIPLES}

\section{A. Activation of the transverse acoustic waves}

The properties of guided acoustic waves in an optical fiber is determined by the cylindrical cladding structure, which permits a discrete sets of longitudinal, radial, torsional and flexural vibrations. The electrostriction-induced acoustic modes present in the forward light scattering are the radial modes $R_{\mathrm{m}}$ and the mixed torsional-radial modes $T R_{2 \mathrm{~m}}$, which define the acoustic waves that propagate radially and 
circumferentially, respectively. The characteristic solutions of $R_{0 \mathrm{~m}}$ modes, $y_{\mathrm{m}}$, could be obtained analytically [2]

$$
\left(1-\alpha^{2}\right) J_{0}(y)-\alpha^{2} J_{2}(y)=0
$$

The cylindrical symmetry of the fibre results in solutions based on the Bessel functions $J_{\mathrm{n}}$, where $\alpha$ is the ratio of the transverse acoustic velocity $V_{s}$ to the longitudinal acoustic velocity $V_{d}$ (e.g. $V_{d}=5968 \mathrm{~ms}^{-1}$ and $V_{s}=3764 \mathrm{~ms}^{-1}$ for fused silica). The eigenfrequencies $f_{\mathrm{m}}$ of the $R_{0 \mathrm{~m}}$ modes are given by

$$
f_{\mathrm{m}}=\mathrm{V}_{\mathrm{d}} y_{\mathrm{m}} /(2 \pi a)
$$

where $a$ is the optical fibre radius. Due to the conservation of momentum, the interaction between guided light inside the fiber core and the transverse acoustic eigenmodes within the cladding structure satisfy the phase matching conditions as in

$$
\begin{gathered}
\mathbf{k}\left(\omega_{\mathrm{p}}\right)=\mathbf{k}\left(\omega_{\mathrm{s}}\right)+\mathbf{K}(\Omega) \\
\omega_{\mathrm{p}}=\omega_{\mathrm{s}}+\Omega
\end{gathered}
$$

where $\mathbf{k}$ and $\mathbf{K}$ are the axial wave vectors of the guided light and the acoustic phonon, respectively, whereas $\omega_{\mathrm{p}}, \omega_{\mathrm{s}}$ and $\Omega$ are the angular frequencies of the pump, Stokes light and the acoustic phonon, respectively. Given $\Delta \mathbf{k}(\omega)=\mathbf{k}\left(\omega_{\mathrm{p}}\right)-\mathbf{k}\left(\omega_{\mathrm{s}}\right)=$ $\mathbf{K}(\Omega)$ and $\Delta \omega=\omega_{\mathrm{p}}-\omega_{\mathrm{s}}=\Omega$, the group velocity of the guided light $v_{\mathrm{g}}$ must be equal to the phase velocity of the acoustic wave $\mathrm{V}_{\mathrm{a}}, v_{\mathrm{g}}=\Delta \omega / \Delta \mathbf{k}(\omega)=\Omega / \mathbf{K}(\Omega)=\mathrm{V}_{\mathrm{a}}$, to have efficient FSBS. The characteristics of the guided transverse acoustic wave in a standard optical fiber is described with the acoustic dispersion diagram $\mathbf{K}(\Omega)$ (Fig. 1(a)). As the axial wave vector $\mathbf{K}$ is close to zero, the phase velocities of the acoustic modes (blue lines in Fig. 1 (a)) are extremely high while their group velocities are negligibly small. In this case, the phase matching condition is relaxed because a wide range of acoustic phase velocities can match with the group velocity of the guided light at a particular acoustic resonance frequency. Since the group velocity of light in optical fiber is close to its phase velocity for standard telecom wavelength, the transverse acoustic waves will propagate with the same phase velocity as the guided light (see Fig. 1(b)).
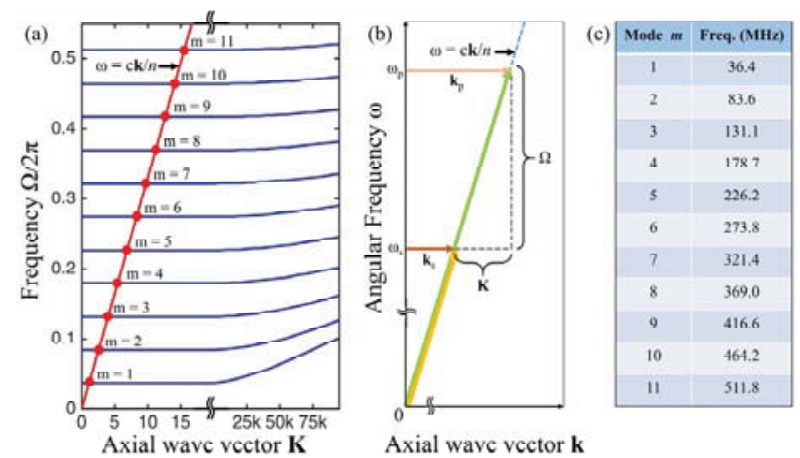

Fig. 1. (a) Dispersion diagram of the transverse acoustic wave (blue) and the guided light (red) in an optical fiber. (b) Phase matching of two coprpagating lights to stimulate FSBS. (c) The resonance frequencies of the acoustic modes.

\section{B. Detecting the response of FSBS}

The stimulated transverse acoustic waves induce small refractive index change in the fiber core because of the photoelastic effect in silica material. This tiny refractive index change can be probed by light in a disparate wavelength. Since the phase velocity of both the probe light and the transverse acoustic wave are almost equal, the probe light follows the same wavefront of the transverse acoustic wave while propagating along the fiber, thus experiencing the refractive index change unique to each point in the traveling transverse acoustic wave, such scenario is equal to being phase-modulated at the acoustic resonance frequency. The phase signal in probe could then be read using an interferometer, which converts the phase change to intensity change that is detectable by a photodiode. We use the widely-proven technique of a Sagnac loop, with CW light in a wavelength different from the pump light, to retrieve the response of the transverse acoustic waves.

\section{EXPERIMENTAL SETUP}

The experimental setup for FSBS activation and detection is shown in Fig. 2. An intensity-modulated optical pulse at $\lambda=$ $1551 \mathrm{~nm}$ serves as the pump source for FSBS. For time-domain measurement technique [3], a short pulse of $1 \mathrm{~ns}$ is shaped from the incoming distributed feedback (DFB) laser by an electrooptic modulator (EOM) whereas for frequency-domain method [4], a long pulse of $500 \mathrm{~ns}$ is first shaped from the laser by a semiconductor optical amplifier (SOA) and subsequently intensity-modulated by an EOM at FSBS frequency where the modulation signal is provided by a vector network analyzer (VNA). The purpose of introducing the long pulse is to suppress the unwanted response due to Kerr cross-phase modulation by means of selectively gating away the affected time duration in the response trace before detection [4]. The generated optical pulse in both techniques is amplified by an Erbium doped fiber amplifier (EDFA) to produce a peak power of around 1 W. A continuous wave from another DFB laser at a disparate wavelength of $1534 \mathrm{~nm}$ is used for probing the induced phase modulation. A tunable bandpass filter (BPF) adjusted to $1534 \mathrm{~nm}$ is placed in the Sagnac loop after the sensing fiber to prevent the pump light from travelling in the rest of the loop, whilst another BPF at the same wavelength is placed before the photodetector to further avoid the pump light from being detected. For time domain measurement, the electrical signal from the photodiode is read by an oscilloscope whereas for the frequency domain measurement, it is read by the VNA, which is synchronized to the pump modulation frequency and configured to sweep across a chosen FSBS resonance peak.

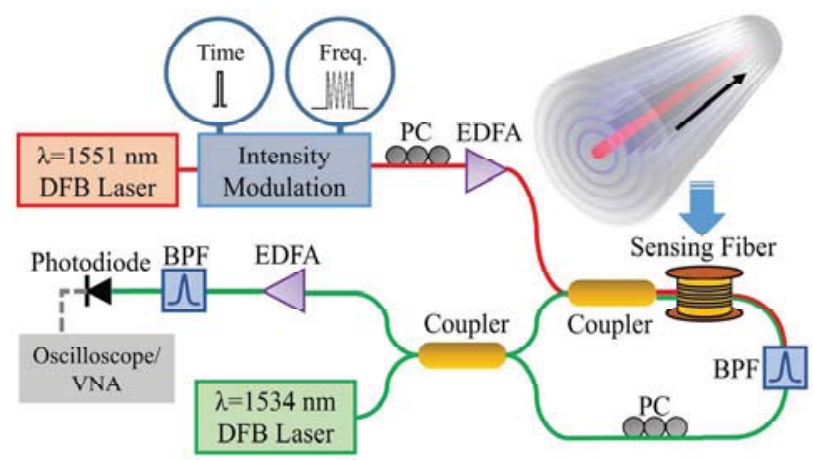

Fig. 2. The experimental setup for activating and detecting FSBS. Red and green paths represent the pump and probe wavelengths, respectively. 


\section{MEASUREMENT OF ACOUSTIC IMPEDANCE}

For direct detection of the surrounding liquid medium, a $30 \mathrm{~m}$ standard optical fiber without the polymer coating is used as the sensing fiber. Fig. 3 shows the response of FSBS when the sensing fiber is exposed to air, measured separately by using time (Fig. 3(a)) and frequency (Fig. 3(b)) domain techniques.
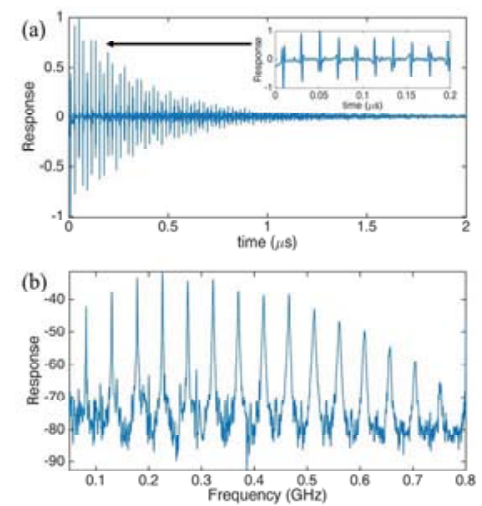

Fig. 3. The FSBS response obtained by (a) time and (b) frequency domain techniques. The sensing fiber is exposed to air.

The acoustic impedance of the surrounding liquid medium is retrieved by finding the acoustic reflectivity at the claddingsurroundings boundary. Because of the large acoustic impedance mismatch between the fiber material and the external medium, part of the transverse acoustic waves are reflected back and trapped inside the transverse cavity. The intensity of successive oscillation cycles reduces exponentially in time due to reflection loss at the fiber boundary and internal loss (viscose damping, structural inhomogeneity, etc.). The reflectivity coefficient, determined by the acoustic impedance mismatch between the fiber material and the surrounding liquid medium, is given by [3]

$$
|r|=\left|Z_{f}-Z_{o}\right| /\left(Z_{f}+Z_{o}\right)
$$

where $Z_{f}$ and $Z_{o}$ are the acoustic impedances of fiber material (silica) and the surrounding liquid medium, respectively. The acoustic impedance of silica is $Z_{f}=13.0 \times 10^{6} \mathrm{~kg} /\left(\mathrm{m}^{2} \mathrm{~s}\right)$. Since the acoustic impedance of air $\left(\sim 409 \mathrm{~kg} /\left(\mathrm{m}^{2} \mathrm{~s}\right)\right)$ is much smaller than silica, the reflection at silica-air boundary is assumed to be perfect and the measured acoustic decay will be caused by the intrinsic loss only. For time domain technique, the reflectivity coefficient is related to the decay rate of each mode by [3]

$$
\frac{1}{\tau_{m}}=\frac{1}{\tau_{\text {int }}}+\frac{1}{t_{r}} \ln \left(\frac{1}{|r|}\right)
$$

where $\tau_{\mathrm{m}}, \tau_{\text {int }}$ and $t_{\mathrm{r}}$ are the decay rate of a measurement, decay rate due to intrinsic loss and the mean duration between consecutive oscillation peaks $\left(t_{\mathrm{r}} \approx 21 \mathrm{~ns}\right)$, respectively. For frequency domain method, the reflectivity coefficient is related to the measured linewidth of a selected mode by [4]

$$
\Delta v_{m}=\Delta v_{i n t}+\frac{\Delta v_{i}}{\pi} \ln \left(\frac{1}{|r|}\right)
$$

where $\Delta v_{\mathrm{m}}, \Delta \mathrm{v}_{\text {int }}$ and $\Delta \mathrm{v}_{\mathrm{i}}$ are the linewidth of a measurement with a surrounding liquid medium, linewidth due to intrinsic loss and the frequency separation between adjacent resonance peaks $\left(\Delta v_{\mathrm{i}} \approx 48 \mathrm{MHz}\right)$, respectively.

As the sensing fiber is immersed in a liquid, the reflectivity at the boundary reduces, resulting in faster decay or broader resonance linewidth. For time-domain technique, the response from a single acoustic mode is spectrally filtered from the detected time response by fast Fourier transform (Fig. 4(a)), from here the decay time $\tau_{\mathrm{m}}$ is retrieved. For frequency-domain technique, the linewidth $\Delta v_{\mathrm{m}}$ is taken from the full-width halfmaximum (FWHM) of the resonance spectrum (Fig. 4(b)). $\tau_{\text {int }}$ and $\Delta v_{\text {int }}$ are retrieved from the fiber surrounded by air. The acoustic impedance of liquids (water and ethanol) are calculated by applying (6) and (7). The results agree well with the standard values (see Table I) therefore indicating the feasibility of directly measuring the mechanical properties of a fiber's external medium with light-induced sound waves.
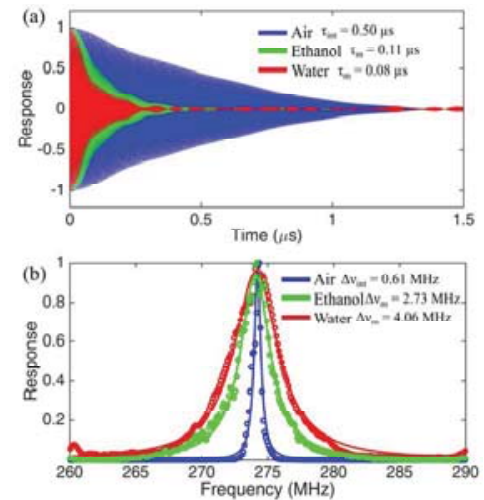

Fig. 4. The detected FSBS response of fiber immersed in liquids. (a) Timedomain method (an acoustic mode is spectrally filtered from the detected time response). (b) Frequency-domain method (the detected resonance spectra).

TABLE I. MEASURED ACOUSTIC IMPEDANCES OF LiquidS

\begin{tabular}{|c|c|c|c|}
\hline Liquid & Time domain & Freq. domain & Standard value* $^{*}$ \\
\hline Ethanol & 0.966 & 0.914 & 0.950 \\
\hline Water & 1.428 & 1.484 & 1.494 \\
\hline
\end{tabular}

*Provided by Onda Corporation, Sunnyvale, CA. All values are in units of $\left(\times 10^{6} \mathrm{~kg} /\left(\mathrm{m}^{2} \mathrm{~s}\right)\right)$

\section{ACKNOWLEDGMENT}

This work was supported by SNSF Grant (200021L-157132).

\section{REFERENCES}

[1] E. Ippen and R. Stolen, "Stimulated Brillouin scattering in optical fibers," Appl. Phys. Lett., vol. 21, no. 11, pp. 539-541, 1972.

[2] R. Shelby, M. Levenson, and P. Bayer, "Guided acoustic-wave Brillouin scattering," Phys. Rev. B, vol. 31, no. 8, p. 5244, 1985.

[3] Y. Antman, A. Clain, Y. London, and A. Zadok, "Optomechanical sensing of liquids outside standard fibers using forward stimulated Brillouin scattering," Optica, vol. 3, no. 5, pp. 510-516, 2016.

[4] D. Chow, M. A. Soto, and L. Thévenaz, "Frequency-domain technique to measure the inertial response of forward stimulated Brillouin scattering for acoustic impedance sensing," in Proceedings of the 25th International Conference on Optical Fiber Sensors, 2017, vol. 10323. 